\title{
Predictor Role of Systemic Inflammation in Ovarian Cancer
}

\author{
Seda Keskin ${ }^{1(\mathbb{D})}$, Duygu Altin ${ }^{1(\mathbb{D})}$ \\ ${ }^{1}$ Obstetrics and Gynecology Department, Medical Faculty, Ordu University, Ordu, Turkey
}

Copyright@ Author(s) - Available online at https://dergipark.org.tr/en/pub/mbsjohs

Content of this journal is licensed under a Creative Commons Attribution-NonCommercial 4.0 International License,

Received: 17 August 2021, Accepted: 26 August 2021, Published online: 31 August 2021

(C) Ordu University Institute of Health Sciences, Turkey, 2021

\begin{abstract}
Objective: The study aims to investigate the diagnostic value of hemogram derived systemic inflammation parameters in ovarian cancer.

Methods: Totally, the study group consisted of 60 patients with suspected ovarian masses who underwent surgery between February 1th, 2020, and May 1th, 2021, in Ordu University Training and Research Hospital. The patients included in the study were divided into two groups according to postoperative histopathological diagnosis, benign group (consisting of 39 patients) and malign group (consisting of 21 patients). The analysis of the receiver operating characteristic (ROC) curve was used to discover the optimal cut-off values of the hemogram derived blood parameters to predict ovarian cancer.

Results: In the cancer group; $85 \%$ of the patients were diagnosed with epithelial ovarian cancer and $62 \%$ at late stage. As compares with benign ovarian mass group, the ovarian cancer group had higher neutrophil counts $(6.67+3.17$ vs $4.64+1.94)(\mathrm{p}=0.006)$, but lower lymphocyte counts $(1.60+0.68$ vs $2.22+0.64)$ $(\mathrm{p}=0.003)$. The high NLR values (cut-off 2.557 ) predict ovarian cancer with $71.4 \%$ sensitivity and $69.2 \%$ specificity (AUC $0.817, \mathrm{p}=0.000, \mathrm{CI}=0.712-0.922$ ). The high $\mathrm{dNLR}$ values (cut- off 1.881 ) also predict cancer with similar sensitivity and specificity as NLR (AUC 0.814, p=0.000, CI 0.708-0.921). Significant cut-off values for the other hemogram derived parameters were $0.26,0.0165,159.66$ and 770.611 for MLR, NPR, PLR and SII, respectively. Additionally, the high values of CA 125 (cut-off 34.45) and CA 15-3 (cutoff 16.4) was founded to be related with ovarian cancer.

Conclusions: This paper revealed that high inflammatory parameters such as NLR and dNLR in patients with ovarian masses are mainly associated with ovarian cancer. In the study, it was emphasized that simple and easily accessible hemogram parameters should be used in addition to tumor biomarkers such as CA 125, CA 15-3, which are routinely used in predicting ovarian cancers. We think that more valuable results will be achieved with comprehensive studies designed prospectively.

Key words: Neutrophil-to-lymphocyte ratio, derived neutrophil-to-lymphocyte ratio, systemic inflammation, ovarian cancer.
\end{abstract}

Suggested Citation: Keskin S, Altin D. Predictor role of systemic inflammation in ovarian cancer Mid Blac Sea Journal of Health Sci, 2021; 7(2):282-288

Address for correspondence/reprints:

E-mail: adesniksek@gmail.com

Seda Keskin

Telephone number: +90 (505) 6273412 


\section{Introduction}

Ovarian cancer is a fairly common malignancy among women. Although, its frequency varies between countries, it ranks third after cervical cancer and uterine corpus cancer. Additionally, ovarian cancer is the most common cause of gynecological cancer-related deaths due to its high incidence and high mortality rate (1). The fact that ovarian cancer is generally asymptomatic at an early stage, and the limited number of sensitive and specific diagnostic markers cause more than two-thirds of the patients to be diagnosed at an advanced stage. Although treatments such as staging or debulking surgery and chemotherapy continue to evolve over the years, the five-year survival rate remains under $30 \%$, due to early metastasis and late diagnosis (2).

Although tumor biomarkers such as CA125 (cancer antigen 125), CA15-3 (cancer antigen 15-3), CA19-9 (cancer antigen 19-9), CEA (carcino embryonic antigen) and AFP (alfa feto protein), which have been used for many years, are in the first place in diagnosis, many other markers have been investigated for early diagnosis of ovarian cancer and to predict prognosis. But which marker should be used is still a matter of debate. Therefore, finding effective markers and using them together with conventional tumor biomarkers is very important in the diagnosis and treatment of ovarian cancer, which is a serious public health problem (3).

Emerging evidence revealed that up to $20 \%$ of the cancers were caused by chronic inflammation, and systemic inflammatory response played a key role in the initiation, invasion, progression and distant metastasis of malignancies. Numerous markers of inflammatory-immune response have been proposed as potential prognostic factors for cancer. Among these inflammation markers hemogram derived ratios such as NLR and dNLR are routinely tested and widely used in clinical practice (4-6).

A newly identified marker, dNLR, calculated using the formula for neutrophil count/non-neutrophil white blood cell count, has been widely investigated to predict prognosis in many types of cancer, including breast, urological, digestive cancers and malignant melanoma $(4,5,7,8)$. However, the diagnostic value of these inflammatory parameters in gynecological (especially ovarian) cancers remains unclear. In the current study, we aimed to reveal the clinical importance of inflammatory parameters in ovarian cancer and to investigate the success in predicting malignancy in ovarian masses.

\section{Methods}

This retrospective, single-center study was conducted between February 1st, 2020, and May 1th, 2021, in Ordu University Training and Research Hospital. The study was approved by the ethics committee of Ordu University Medical Faculty.

We retrospectively analyzed 96 patients with suspected ovarian mass who underwent surgery. Nonconfirmed PCR negative cases, even if they were symptomatic or with a history of contact, were excluded from the study. Pregnancy, age $<18$ years, acute inflammation, blood disease and smokers were excluded from cohort. 11 covid-19 suspected/positive, 18 smokers, 4 tubo-ovarian abscess/pelvic infections, 2 blood diseases, 1 pregnant patient were excluded from the study. As a result, a cohort was formed with 60 patients who met the study criteria.

Patients with ovarian masses included in the study were divided into two groups according to their postoperative histopathological diagnosis. Thence, there was benign group (consisting of 39 patients) and malign group (consisting of 21 patients).

As a routine protocol, hemogram and tumor biomarkers (Ca 125, Ca 15-3, Ca 19-9, CEA, AFP) tests were applied to each patient for preoperative evaluation. Blood samples were taken from all patients upon admission before any treatment began. Primary objective of the study is whether hematological parameters and some inflammatory indices derived from hematological parameters may be used in ovarian cancer patients pre-operatively as simple screening.

Hematologic indices, hematologic ratios and tumor biomarkers were presented in Table 1. These hematological indices were calculated as NLR, which is the ratio between the count of neutrophils $(\times 109$ cells $/ \mathrm{L})$ and the count of lymphocytes $(\times 109$ cells/L), dNLR is neutrophils/(white blood cellsneutrophils), PLR is the ratio between the count of platelets $(\times 1011$ cells $/ \mathrm{L})$ and the count of lymphocytes $(\times 109$ cells/L) and the SII is defined as the counts of neutrophils $(\times 109$ cells/L) multiplied by the counts of platelets $(\times 1011$ cells/L) and divided by the count of lymphocytes $(\times 109$ cells/L), NPR is the ratio between the count of neutrophils $(\times 109$ cells $/ \mathrm{L})$ and the count of platelets $(\times 1011$ cells $/ \mathrm{L})$.

\section{Statistical analysis}

For analyzing the results of the study, IBM SPSS version 20 (SPSS Inc., Chicago, IL, USA) program was used. Analyzes were carried out in a 95\% $(\mathrm{p}=0.05)$ confidence interval. Because the study period is short and so study population is limited and 
retrospective design of the study, sample size is not calculated. Descriptive statistical methods and comparative statistics had been used in the study. Descriptive data derived from the study were presented as mean \pm standard deviation. The normality distribution of numerical variables was studied with the Kolmogorov-Smirnov and the Shapiro-Wilks tests. The independent samples t-test was used for numerical variables with normal distribution and Mann-Whitney $U$ test was used for those which not distributed normally. The analysis of the receiver operating characteristic (ROC) curve was used to discover the optimal cut-off values of the hemogram derived blood parameters to predict ovarian cancer. AUC was interpreted as excellent if 0.9 -AUC-1, good if 0.8 -AUC-0.9, moderate if 0.7 AUC-0.8, poor if 0.6 -AUC-0.7, and failed if 0.5 AUC-0.6.

\section{Results}

Totally, sixty women who were operated for an ovarian mass were included in the retrospective, cohort study. Twenty-one had pathologically confirmed ovarian cancer and thirty-nine were diagnosed benign ovarian cysts.

The demographic and clinical characteristics are presented in Table 1. The age range of patients was 18-80 years. Although the mean age was lower in the benign group $(42.03+13.6)$, no statistically significant difference was observed with the mean age of the malignant group $(49.1+13.6) \quad(p=0.661)$. Additionally, there was no significant difference in body mass indexes between the groups $(23.6+4.4$ vs $22.7+3.4)(\mathrm{p}=0.342)$.

In the cancer group, eighteen women (85.75) had epithelial ovarian cancer, while three patients had a non-epithelial tumor subtype. FIGO (International Federation of Gynecology and Obstetrics) staging was performed in cases with ovarian cancer. FIGO stages were as follows; 1 - six patients, 2 - two patients, 3- eleven patients, 4- 2 patients. Approximately $62 \%$ of patients had advanced (stage 3-4) ovarian cancer.

The hematologic and tumor biomarkers of the study group are presented in Table 2. As compares with benign ovarian mass group, the ovarian cancer group had significantly higher neutrophil counts $(6.67+3.17$ vs $4.64+1.94) \quad(\mathrm{p}=0.006)$, but lower lymphocyte counts $(1.60+0.68$ vs $2.22+0.64)$ $(\mathrm{p}=0.003)$. Accordingly, we found that many hemogram derived parameters, especially NLR (neutrophil-to-lymphocyte ratio) and dNLR (derived neutrophil-to-lymphocyte ratio), increased due to systemic inflammation in the ovarian cancer group. In addition, we found statistically significantly higher tumor biomarkers CA 125 and CA 15-3 in the ovarian cancer group. On the other hand, no significant difference between the groups in terms of CA 19-9, CEA and AFP values was detected.

ROC curves were made to compare the diagnostic utility of tumor biomarkers (CA 125, CA 15-3, CA 19-9, CEA, AFP) and hemogram derived ratios (NLR, dNLR, MLR, PLR, NPR, SII) in diagnosing ovarian cancer. ROC graphics and Area Under the Curve (AUC) values are presented in Figure 1 and Table 3.

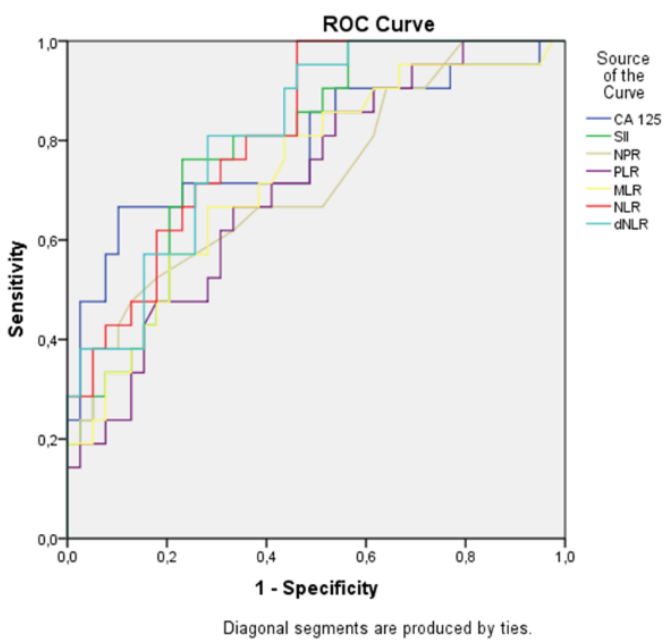

Figure 1. ROC graphics

We found that the high NLR and dNLR had an impact in ovarian cancer. The high NLR values (cutoff 2.557) predict ovarian cancer with $71.4 \%$ sensitivity and $69.2 \%$ specificity (AUC 0.817, $\mathrm{p}=0.000, \mathrm{CI}=0.712-0.922)$. The high dNLR values (cut- off 1.881) also predict cancer with similar sensitivity and specificity as NLR (AUC 0.814, $\mathrm{p}=0.000$, CI 0.708-0.921). Significant cut-off values for the other hemogram derived parameters were $0.26,0.0165,159.66$ and 770.611 for MLR, NPR, PLR and SII, respectively. Additionally, the high values of CA 125 (cut-off 34.45) and CA 15-3 (cutoff 16.4) was founded to be related with ovarian cancer. 
Table 1. The demographic and clinical characteristics of the study group

\begin{tabular}{lccc}
\hline Characteristics & Ovarian cancer $(\mathrm{n}=21)$ & Benign ovarian masses $(\mathrm{n}=39)$ & P value \\
\hline Age & $49.10 \pm 13.68$ & $42.03 \pm 13.03$ & 0.661 \\
\hline BMI & $23.6 \pm 4.4$ & $22.7 \pm 3.4$ & 0.342 \\
\hline Tumor subtype & & & \\
\hline Epithelial & 18 & & \\
\hline Non-epithelial & 3 & & \\
\hline FIGO stage & & & \\
\hline I & 6 & \\
\hline II & 2 & \\
\hline III & 11 & & \\
\hline IV & 2 & & \\
\hline
\end{tabular}

Abbreviations: BMI, body mass index; FIGO, International Federation of Gynecology and Obstetrics

Table 2. Hematologic and tumor biomarkers of the study group **

\begin{tabular}{|c|c|c|c|}
\hline Biomarker & Ovarian cancer $(\mathrm{n}=21)$ & Benign ovarian masses $(n=39)$ & P value \\
\hline WBC & $9.08 \pm 3.26$ & $7.62 \pm 2.41$ & 0.140 \\
\hline $\mathrm{HB}$ & $11.95 \pm 1.67$ & $11.96 \pm 1.55$ & 0.843 \\
\hline NEU & $6.77 \pm 3.17$ & $4.64 \pm 1.94$ & $0.006^{*}$ \\
\hline LYM & $1.60 \pm 0.68$ & $2.22 \pm 0.64$ & $0.003 *$ \\
\hline MONO & $0.54 \pm 0.21$ & $0.53 \pm 0.16$ & 0.798 \\
\hline EOS & $0.11 \pm 0.09$ & $0.20 \pm 0.26$ & 0.158 \\
\hline BASO & $0.034 \pm 0.022$ & $0.031 \pm 0.020$ & 0.721 \\
\hline MCV & $85.22 \pm 6.82$ & $84.14 \pm 7.40$ & 0.369 \\
\hline $\mathrm{MCH}$ & $27.32 \pm 2.66$ & $27.12 \pm 3.02$ & 0.969 \\
\hline MCHC & $32.02+1.06$ & $32.17 \pm 1.53$ & 0.090 \\
\hline PCT & $0.28 \pm 0.04$ & $0.30 \pm 0.08$ & 0.309 \\
\hline PDW & $11.10 \pm 2.24$ & $10.67 \pm 1.94$ & 0.721 \\
\hline RDW & $44.78 \pm 7.90$ & $41.69 \pm 9.62$ & 0.117 \\
\hline PLT & $287.47 \pm 55.97$ & $310.48 \pm 84.82$ & 0.120 \\
\hline NLR & $5.81 \pm 6.22$ & $2.19 \pm 1.06$ & $0.000 *$ \\
\hline dNLR & $3.75 \pm 3.58$ & $1.58 \pm 0.64$ & $0.000 *$ \\
\hline MLR & $0.37 \pm 0.18$ & $0.25 \pm 0.10$ & $0.003 *$ \\
\hline PLR & $226.44 \pm 159.35$ & $148.18 \pm 52.58$ & $0.008 *$ \\
\hline NPR & $0.024 \pm 0.015$ & $0.015 \pm 0.006$ & $0.005^{*}$ \\
\hline MPVPR & $0.035 \pm 0.009$ & $0.034 \pm 0.012$ & 0.281 \\
\hline LYM*PLT & $474.02+283.50$ & $705.34+303.79$ & $0.003 *$ \\
\hline RDWPR & $0.16 \pm 0.04$ & $0.014 \pm 0.05$ & 0.072 \\
\hline SII & $1646.38 \pm 1728.93$ & $710.90+503.99$ & $0.000^{*}$ \\
\hline CA 125 & $295.06+408.20$ & $37.11+66.12$ & $0.000^{*}$ \\
\hline CA 15-3 & $41.43 \pm 40.68$ & $12.68 \pm 6.09$ & $0.000 *$ \\
\hline CA 19-9 & $971.89 \pm 4359.99$ & $15.21 \pm 27.35$ & 0.138 \\
\hline CEA & $8.45 \pm 21.3$ & $1.80 \pm 2.05$ & 0.055 \\
\hline AFP & $11.42 \pm 44.36$ & $1.84 \pm 1.21$ & 0.710 \\
\hline \multicolumn{4}{|c|}{$\begin{array}{l}\text { Abbreviations: WBC, white blood cell; HB, hemoglobin; NEU, neutrophil; LYM, lymphocyte; MONO, monocyte; EOS, eosinophil; BASO, basophi } \\
\text { MCV, mean corpuscular volume; MCH, mean corpuscular hemoglobin; MCHC, mean corpuscular hemoglobin concentration; PCT, platecrit; PDW, } \\
\text { platelet distribution width; RDW, red cell distribution width; PLT, platelet; NLR, neutrophil-to-lymphocyte ratio; dNLR, derived neutrophil-to- } \\
\text { lymphocyte ratio; MLR, monocyte-to-lymphocyte ratio; PLR, platelet-to-lymphocyte ratio; NPR, neutrophil-to-platelet ratio; MPVPR, mean platelet } \\
\text { volume-to-platelet ratio; LYM*PLT, lymphocyte*platelet; RDWPR, red cell distribution width-to-platelet ratio; SII, systemic immune inflammation } \\
\text { index; CA 125, cancer antigen 125; Ca 15-3, cancer antigen 15-3; CA 19-9, cancer antigen 19-9; CEA, carcino embryonic antigen; AFP, alfa feto } \\
\text { protein } \\
\text { *statically significant } \\
\text { **values are given as mean + standard deviation }\end{array}$} \\
\hline
\end{tabular}

Table 3: Area Under the Curve (AUC) values

\section{Test Result}

Variable(s)
Area Std. Error ${ }^{\mathrm{a}}$ Asymptotic Sig. ${ }^{\mathrm{b}}$ Asymptotic $95 \%$ Confidence Interval Cut off value Sensitivity Spesificity Lower Bound Upper Bound Hemogram derived ratios

\begin{tabular}{|c|c|c|c|c|c|c|c|c|}
\hline \\
\hline NLR & .817 & .054 & $.000^{*}$ & .712 & .922 & 2.557 & 71.2 & 69.2 \\
\hline dNLR & .814 & .054 & $.000 *$ & .708 & .921 & 1.881 & 71.4 & 71.8 \\
\hline MLR & .732 & .068 & $.003 *$ & .598 & .866 & 0.26 & 66.7 & 61.5 \\
\hline NPR & .720 & .070 & $.005^{*}$ & .582 & .858 & 0.016 & 66 & 62 \\
\hline PLR & .709 & .068 & $.008 *$ & .576 & .842 & 159.6 & 61.9 & 69.2 \\
\hline SII & .794 & .057 & $.000 *$ & .681 & .906 & 770.6 & 76.2 & 76.9 \\
\hline \multicolumn{9}{|c|}{ Tumor biomarkers } \\
\hline CA 125 & .789 & .067 & $.000 *$ & .657 & .921 & 37.4 & 71.4 & 74.4 \\
\hline CA 15-3 & .817 & .064 & $.000 *$ & .693 & .942 & 17.4 & 76.2 & 74.4 \\
\hline
\end{tabular}

a. Under the nonparametric assumption

b. Null hypothesis: true area $=0.5$

*Statically significant 


\section{Discussion}

The systemic inflammatory response plays a significant role in tumor development and progression. Although the genetic predisposition in the development of cancer cannot be denied, many studies have shown that inflammation caused DNA damage and excessive production of cytokines (including IL-2, IL-6, TNF-alfa and VEGF) triggered the initiation and progression of cancer. In addition, it has been determined that inflammation inhibits the apoptosis of DNA damaged cells and increases angiogenesis that helps growth of tumor tissue (9-11).

Although some studies were conflicting, recent evidence showed that systemic inflammatory response markers such as NLR, dNLR, MLR, NPR, PLR and SII were associated with prognosis of various cancer. In a meta-analysis of 6585 patients published in 2019 investigating urological cancers, high dNLR values were associated with decreased cancer-specific survival in renal cell carcinoma, prostate cancer, and urothelial cancers (8). In a study investigating the clinical outcome of patients diagnosed with metastatic gallbladder cancer, it was shown that dNLR and CEA values predict a better prognosis when used together (12).

In a meta-analysis report investigating poor prognosis in 10599 breast cancer patients, high dNLR value was found to be associated with poorer overall and recurrence-free survival (7). In another breast cancer study, NLR, dNLR, PLR values were found to be associated with both disease-specific and diseasefree survival. Especially in patients with breast cancer with high PLR values, more lymph node metastases were detected (6). Furthermore, the relationship between dNLR and cancer survival has been demonstrated in many cancers such as malignant melanoma and digestive cancers $(4,5)$.

In the literature, studies investigating hematological parameters and gynecological cancers are insufficient. In a study investigating cervical cancer prognosis, NLR, dNLR, and PLR were associated with lymph node metastasis, recurrencefree and overall survival (13). In a meta-analysis of data from 3390 patients diagnosed with endometrial cancer, high pretreatment NLR and PLR values were founded to be associated with poor prognosis (14). In two studies examining lymph node metastasis in endometrial cancer, it was shown that hemogram parameters predict lymph node metastasis $(15,16)$. In three other endometrial cancer studies, hemogram parameters showing systemic inflammation were shown to be associated with cancer stage, overall survival, and lymphovascular-myometrial-cervical invasion (17-19).

In studies on ovarian cancer, it has been shown that high NLR and dNLR values worsen the prognosis and can be used together with tumor biomarkers such as CA 125 to predict ovarian cancer (20-22).

In our study, the hemogram derived parameters (NLR, dNLR, MLR, NPR, PLR, LYM*PLT and SII) revealing systemic inflammation in the ovarian cancer group was significantly higher. Although tumor biomarkers (especially CA 125) are in widespread use, their low sensitivity and specificity has always been a problem. In the current study we also found that CA 125 and CA 15-3 predicted ovarian cancer with an average sensitivity and specificity of $70-76 \%$. Therefore, we think that the use of NLR and dNLR ratio and tumor biomarkers together will be more useful in predicting ovarian cancer.

The limitations of the study were the retrospective design and small cohort of the study. In the future, there is a need for studies with larger patient numbers in which the subtypes of benign ovarian masses are also examined in detail. Being a single center study and evaluation of the patients by the same team overall period are other advantages of the study. On the other hand, examining many hemogram and hemogram derived parameters together with tumor biomarkers is the main factor that strengthens our study.

\section{Conclusion}

Today, the importance of systemic inflammation in cancer development is frequently studied. In support of this, we found the hemogram derived parameters (NLR, dNLR, MLR, NPR, PLR, LYM*PLT and SII) in the ovarian cancer group was significantly higher than in the benign ovarian mass.

In the study, it was emphasized that simple and easily accessible hemogram parameters should be used in addition to tumor biomarkers such as CA 125 , CA 15-3, which are routinely used in predicting ovarian cancers. We think that more valuable results will be achieved with comprehensive studies designed prospectively. 
Ethics Committee Approval: This study was conducted with the approval of the ethics committee of Ordu University Faculty of Medicine, NonInvasive Clinical Research Ethics Committee. (Ethics Committee date and Decision no: 12.08.2021 2021/186)

Peer-review: Externally peer-reviewed.

\section{Author Contributions:}

Idea, Design, Audit, Data Collection and/or Processing, Analysis and/or Interpretation: Writing, S.K, D.A

Conflict of Interest: The authors certify that they have no financial or non-financial interest in the subject matter or materials discussed in this manuscript.

Financial Disclosure: The author declared that this study hasn't received no financial support.

\section{References}

1. Wild CP, Weiderpass E, Stewart BW, editors. World Cancer Report: International Agency for Research on Cancer. World Health Organization. ISBN (PDF) 978-92-832-0448-0

2. Berek JS, Crum C, Friedlander M. Cancer of the ovary, fallopian tube, and peritoneum. Int $\mathrm{J}$ Gynaecol Obstet. 2015;131(2):111-22.

3. Wang HP, Wu HY, Wang Y, Wang L. Combined detection of tumor markers and serum inflammatory factors in the diagnosis and treatment of gynecologic oncology. J Biol Regul Homeost Agents. 2017;31(3):691-5.

4. Liu F, Luo H, Zhu Z, Zhu P, Huang J. Prognostic significance of peripheral blood-derived neutrophil/lymphocyte ratio in patients with digestive cancer. J Cell Physiol. 2019;234(12):22775-86.

5. Afzal MZ, Sarwar T, Shirai K. Prognostic Significance of Hematological Indices in Malignant Melanoma Treated With Immune Checkpoint Inhibitors. J Immunother. 2019;42(7):251-64.

6. Cho U, Park HS, Im SY, Yoo CY, Jung JH, Suh $\mathrm{YJ}$, et al. Prognostic value of systemic inflammatory markers and development of a nomogram in breast cancer. PLoS One. 2018;26;13(7):e0200936.

7. Duan J, Pan L, Yang M. Preoperative elevated neutrophil-to-lymphocyte ratio (NLR) and derived NLR are associated with poor prognosis in patients with breast cancer: A meta-analysis. Medicine (Baltimore). 2018;97(49):e13340.
8. Su S, Liu L, Li C, Zhang J, Li S. Prognostic role of pretreatment derived neutrophil to lymphocyte ratio in urological cancers: A systematic review and meta-analysis. Int J Surg. 2019;72:146-53.

9. Grivennikov SI, Greten FR, Karin M. Immunity, inflammation, and cancer. Cell. 2010;19;140(6):883-99.

10.Hagerling C, Casbon AJ, Werb Z. Balancing the innate immune system in tumor development. Trends Cell Biol. 2015;25(4):214-20.

11.Lin EY, Pollard JW. Role of infiltrated leucocytes in tumour growth and spread. $\mathrm{Br} \mathrm{J}$ Cancer. 2004;90(11):2053-8.

12. Du JH, Lu J. Circulating CEA-dNLR score predicts clinical outcome of metastatic gallbladder cancer patient. J Clin Lab Anal. 2019;33(2):e22684.

13.Chen L, Zhang F, Sheng XG, Zhang SQ, Chen YT, Liu BW. Peripheral platelet/lymphocyte ratio predicts lymph node metastasis and acts as a superior prognostic factor for cervical cancer when combined with neutrophil: Lymphocyte. Medicine (Baltimore). 2016;95(32):e4381.

14.Ni L, Tao J, Xu J, Yuan X, Long Y, Yu N, et al. Prognostic values of pretreatment neutrophil-tolymphocyte and platelet-to-lymphocyte ratios in endometrial cancer: a systematic review and metaanalysis. Arch Gynecol Obstet. 2020;301(1):25161.

15. Kadan Y, Calvino AS, Katz A, Katz S, Moore RG. Predictors for lymph nodes involvement in low risk endometrial cancer. J Obstet Gynaecol. 2017;37(4):514-8.

16. Aoyama T, Takano M, Miyamoto M, Yoshikawa T, Kato K, Sakamoto T, et al. Pretreatment Neutrophil-to-Lymphocyte Ratio Was a Predictor of Lymph Node Metastasis in Endometrial Cancer Patients. Oncology. 2019;96(5):259-67.

17. Temur I, Kucukgoz Gulec U, Paydas S, Guzel AB, Sucu M, Vardar MA. Prognostic value of preoperative neutrophil/lymphocyte ratio, monocyte count, mean platelet volume, and platelet/lymphocyte ratio in endometrial cancer. Eur J Obstet Gynecol Reprod Biol. 2018;226:259.

18. Bacanakgil BH, Kaban I, Unal F, Guven R, Sahin E, Yildirim SG. Predictive Value of Hematological Inflammatory Markers in Endometrial Neoplasia. Asian Pac J Cancer Prev. 2018;25;19(6):1529-32. 
19.Cong R, Kong F, Ma J, Li Q, Wu Q, Ma X. Combination of preoperative neutrophillymphocyte ratio, platelet-lymphocyte ratio and monocyte-lymphocyte ratio: a superior prognostic factor of endometrial cancer. BMC Cancer. 2020;24;20(1):464.

20.Wu YY, Qin YY, Qin JQ, Zhang X, Lin FQ. Diagnostic value of derived neutrophil-tolymphocyte ratio in patients with ovarian cancer. J Clin Lab Anal. 2019;33(4):e22833.

21.Chen L, Wang X, Shu J, Xu S, Wu Q, Yu Y. Diagnostic value of serum D-dimer, CA125, and neutrophil-to-lymphocyte ratio in differentiating ovarian cancer and endometriosis. Int J Gynaecol Obstet. 2019;147(2):212-8.

22.Zhao Z, Zhao X, Lu J, Xue J, Liu P, Mao H. Prognostic roles of neutrophil to lymphocyte ratio and platelet to lymphocyte ratio in ovarian cancer: a meta-analysis of retrospective studies. Arch Gynecol Obstet. 2018;297(4):849-57. 